ISSN: $2637-4609$

\title{
The Carbonyl Groups as Scaffolds in Coordination Chemistry
}

John Markopoulos*

Department of chemistry, National and Kapodistrian University of Athens, Greece

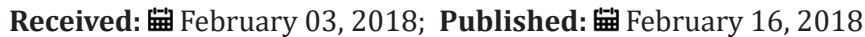

*Corresponding author: John Markopoulos, Department of chemistry, National and Kapodistrian University of Athens, 15771, Greece, Email: jmmarko@chem.uoa.gr

\section{Introduction}

Carbonyl compounds are of paramount importance in the formation of $\mathrm{C}-\mathrm{C}$ bonds and metal-ion control of their reactivity offers a way to promote, inhibit, or control such reactions [1]. The coordination of the oxygen atom of a carbonyl compound to a metal center is likely to modify the reactivity of that group. Products formally derived by attack at the carbonyl group may arise either by direct attack at the carbon atom or by attack at the metal followed by attack at carbon by the coordinated nucleophile. There has been a debate over the relative importance of these two competing mechanisms in biological and biomimetic systems. These two mechanisms may well both be operative in some cases. The principal reactions of carbonyl compounds are of two types: The first type is that in which the nucleophile reacts with the electrophilic carbon atom of a carbonyl group to generate a new tetrahedral center (Figure 1).

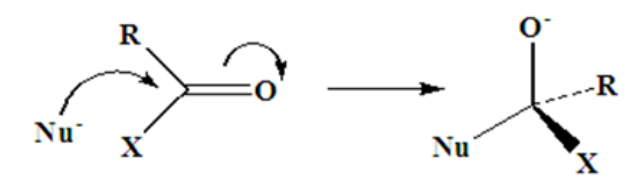

Figure 1: The first type is that in which the nucleophile reacts with the electrophilic carbon atom of a carbonyl group to generate a new tetrahedral center.

The fate of this tetrahedral intermediate is depended upon the nature of the substituents $\mathrm{R}$ and $\mathrm{X}$ and upon the incoming nucleophile. If $\mathrm{X}$ - is a better leaving group than $\mathrm{Nu}-$, the overall reaction is a nucleophilic substitution of $\mathrm{X}$ by $\mathrm{Nu}$, with the resultant loss of $\mathrm{X}$ - (Figure 2). In contrast, if $\mathrm{X}$ is not a better leaving group than $\mathrm{Nu}$ - then either $\mathrm{Nu}$-is lost (with no overall resulting reaction) or a substituted alcohol may be formed by reaction of the tetrahedral anion with a proton (Figure 3). The second type of reaction associated with carbonyl compounds, involves the generation of nucleophile enol or enolate ion. Although the conversion of a ketone to the tautomeric enol does not necessarily involve any other species, the generation of an enolate requires a base (Figure 4). The carbon atom of the enol or enolate is nucleophilic and may undergo facile reation with electrophiles.

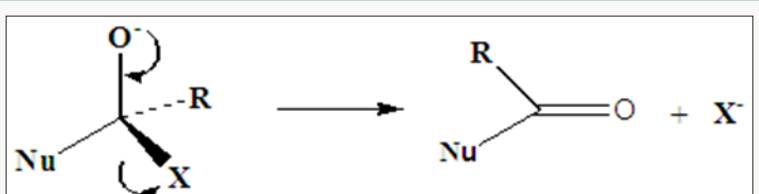

Figure 2: If $\mathrm{X}$ - is a better leaving group than $\mathrm{Nu}^{-}$, the overall reaction is a nucleophilic substitution of $\mathrm{X}$ by $\mathrm{Nu}$, with the resultant loss of $\mathrm{X}-$.

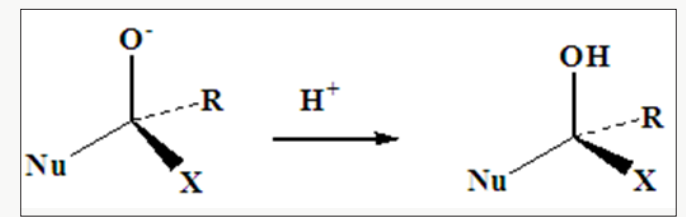

Figure 3: In contrast, if $\mathrm{X}$ is not a better leaving group than $\mathrm{Nu}$ - then either $\mathrm{Nu}$-is lost (with no overall resulting reaction) or a substituted alcohol may be formed by reaction of the tetrahedral anion with a proton. 


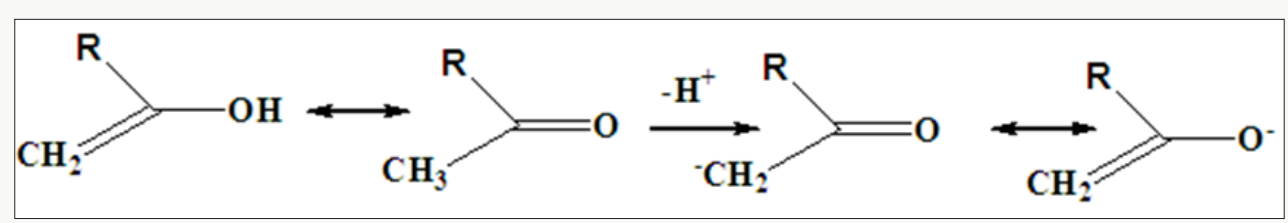

Figure 4: Although the conversion of a ketone to the tautomeric enol does not necessarily involve any other species, the generation of an enolate requires a base.

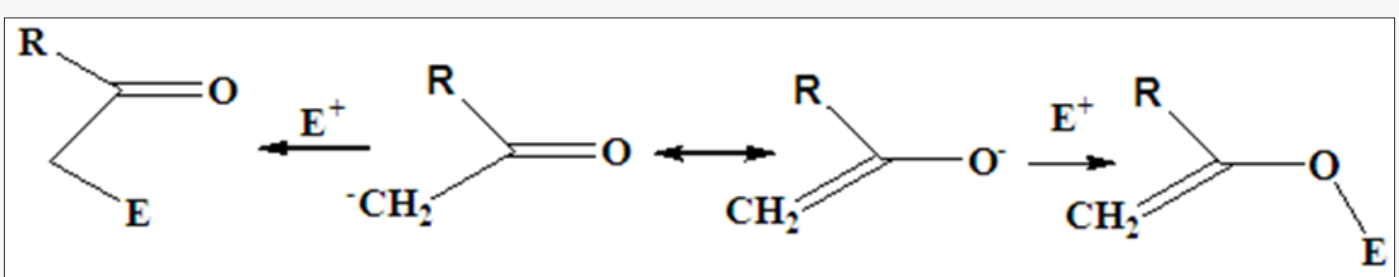

Figure 5: The carbon atom of the enol or enolate is nucleophilic and may undergo facile reation with electrophiles. In other cases, the electrophile can react with oxygen atom.

In other cases, the electrophile can react with oxygen atom (Figure 5). The relative importance of these two modes of attack depends upon the nature of electrophile and, to a lesser extent, upon the specific enolate. Both of these fundamental types of reactivity of a carbonyl compound may be modified by coordination to metal ions. The polarization effect means that the electrophilic character of a carbonyl carbon atom may be increased by coordination of the oxygen to a metal ion, and so, any reactions in which the rate- determining step is nucleophilic attack upon this site might be accelerated. Similarly, the presence of an electropositive metal coordinated to the oxygen atom might favour enolisation and accelerate reactions in which enolisation or reactions of an enolate are rate-determining. The pKa of the enol will also be sensitive to coordination of the oxygen, and generation of the enolate may be facilitated.<smiles></smiles>

Figure 6: The enol form due to the formation of the distinct resonance structure as a six-membered ring.

A case example of the above status is the study of diketones and especially the $\beta$-diketones. $\beta$-diketone compounds, whose simplest and the most widely known member is pentane-2,4-dione (acetylacetone) have a number of very interesting and specific properties due to their structure (the presence of two carbonyl groups separated by one carbon atom). Their crucial feature is ketoenol tautomerism, the presence of the ketone and enol forms in equilibrium. The equilibrium in the case of $\beta$-diketones is strongly shifted towards the enol form due to the formation of the distinct resonance structure as a six-membered ring (Figure 6). Keto-enol equilibrium is affected by a number of other factors with the most important being solvent polarity and the presence and properties of subsituents (both terminal ones and those in methylene group).

The capacity to form stable complexes with most metals is a direct consequence of the occurrence of such compounds in the enol form. Owing to their properties, $\beta$-diketones have been used in both in science and industry. They also were used in healthcare both as active pharmaceutical ingredients or substrates for the manufacture of medicine and cosmetic additives which reduce the detrimental effects of UV radiation of the skin. Furthermore they are important for chemical analysis in which they are used for sample concentration, for air pollution monitoring, and for environmental protection [2-4].

\section{References}

1. Hay RW (1987) In Comprehensive Coordination Chemistry. Wilkinson G, Gillard RD, McCleverty JA, (Eds.), Oxford, Pergamon, Turkey, p. 6.

2. Gullen WR, Wickenheiser EB (1989) Rhodium (I) complexes of $\beta$-diketonates and related ligands as hydrosilylation catalysts. J Organomet Chem 370(1-3): 141-154.

3. Lewis FD, Miller AM, Salvi DG (1995) Spectroscopy and Photochemistry of Nickel(II), Palladium(II) and Platinum(II) $\beta$-diketonates. Inorg Chem 34(12): 3173-3181.

4. Meshkowa SB (2000) The dependence of the luminescence intensity of lanthanide complexes with $\beta$-diketonates on the ligand form. Journal of Fluoresence 10(4): 333-337. 
(C) (1) This work is licensed under Creative To Submit Your Article Click Here: Submit Article DOI: 10.32474/AOICS.2018.01.000119

\section{AOICS}

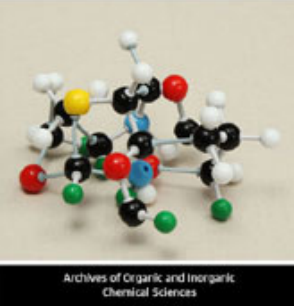

\section{Archives of Organic and Inorganic} Chemical Sciences

Assets of Publishing with us

- Global archiving of articles

- Immediate, unrestricted online access

- Rigorous Peer Review Process

- Authors Retain Copyrights

- Unique DOI for all articles 